\title{
Highly Efficient Broadband Silicon-on-Insulator Grating Couplers for the Short Wave Infrared Wavelength Range
}

\author{
B. Kuyken ${ }^{1}$, N. Hattasan ${ }^{1}$, D. Vermeulen ${ }^{1}$, S. Selvaraja ${ }^{1}$, W. Bogaerts ${ }^{1}$, W. Green ${ }^{2}$, R. Baets ${ }^{1}$, G. Roelkens ${ }^{1}$ \\ ${ }^{I}$ Photonics Research Group, Department of Information Technology, Ghent University - imec, Ghent B-9000, Belgium \\ ${ }^{2}$ IBM T. J. Watson Research Center, 1101 Kitchawan Road, Yorktown Heights, NY 10598, USA. \\ Tel.:+32 (0)9 32-9-264-3335.Email: Bart.Kuyken@intec.ugent.be \\ Abstract: We demonstrate broadband silicon-on-insulator fiber-to-chip grating couplers for the \\ short wave infrared region. The devices show a peak coupling loss of $-5.2 \mathrm{~dB}$ at $2150 \mathrm{~nm}$ and a 3 \\ $\mathrm{dB}$ bandwidth of $160 \mathrm{~nm}$. \\ (C) 2011 Optical Society of America \\ OCIS codes: (130.0130) Integrated optics; (130.3120) Integrated optics devices.
}

\section{Introduction}

Over the last decade the silicon-on-insulator platform has become the leading platform for integrated photonics. The progress was mainly driven by applications for telecom, since silicon is transparent at telecommunication wavelengths. It has lead to a whole range of integrated photonics devices for telecom applications such as arrayed waveguide gratings, planar concave gratings and sharp add/drop filters [1]. However, the platform is transparent up to $3.5 \mu \mathrm{m}$, limited by the absorption of the oxide buffer layer [2] and has recently been proposed as a platform for these longer wavelengths. This region is of enormous interest for spectroscopic applications, since many molecules have distinct absorption bands in this region [3]. Moreover, this wavelength range will not only enable a whole set of new optical sensing components, but it has also shown to be of great interest in a nonlinear optics context. Working at these longer wavelengths reduces the parasitic nonlinear absorption in silicon, the two photon absorption, enormously. Without the two photon absorption we can fully benefit from the record nonlinear parameters obtained through the combination of the high linear index leading to high confinement and the high nonlinear index of silicon. Indeed recent demonstrations have shown highly efficient nonlinear optical devices enabling high parametric gain [4], wavelength conversion [4, 5] and efficient supercontinuum generation [6].

However, interfacing with chips containing these new mid-infrared devices has been very limited so far, as it is mostly achieved by coupling lensed fibers directly to the waveguides. Low coupling efficiencies of around $-10 \mathrm{~dB}$ are achieved as a result of the bad overlap between the spot of these lensed fibers and the waveguide mode. In this paper we propose diffractive grating couplers optimized for this new wavelength region. We achieve coupling losses as low as $-5.2 \mathrm{~dB}$ and a $3 \mathrm{~dB}$ bandwith of $160 \mathrm{~nm}$. The central wavelength of the grating couplers is $2150 \mathrm{~nm}$, a wavelength at the edge of the two-photon absorption cut-off wavelength and well covering the finger print absorption bands of many molecules (e.g. glucose).

\section{Design of the fiber grating coupler}

The Bragg condition describes the relation of the incident and diffracted waves as shown in equation 1

$$
k_{\mathrm{z}}=\beta-\mathrm{K}
$$

where $\beta=\frac{2 \pi}{\lambda} n_{\text {eff }}$ is the propagation constant of the guided mode and $K=\frac{2 \pi}{\Lambda}$, with $\Lambda$ the period of the grating. $k_{z}=\frac{2 \pi}{\lambda} \sin \theta$ is the projected diffracted wave vector, with $\theta$ the angle of the phase fronts of the diffracted field with respect to the silicon surface (and hence the angle of the optical fiber with respect to the surface normal for optimal fiber coupling). From the Bragg condition, the period of the grating coupler is calculated for a given peak wavelength. However, the coupling efficiency depends mostly on the directionality which is a function of waveguide thickness, etch depth and thickness of the silicon overlay. The coupling efficiency also depends on the matching of the coupling strength of the grating coupler and the fiber mode. A schematic cross-section of the grating coupler is shown in Fig.1. 


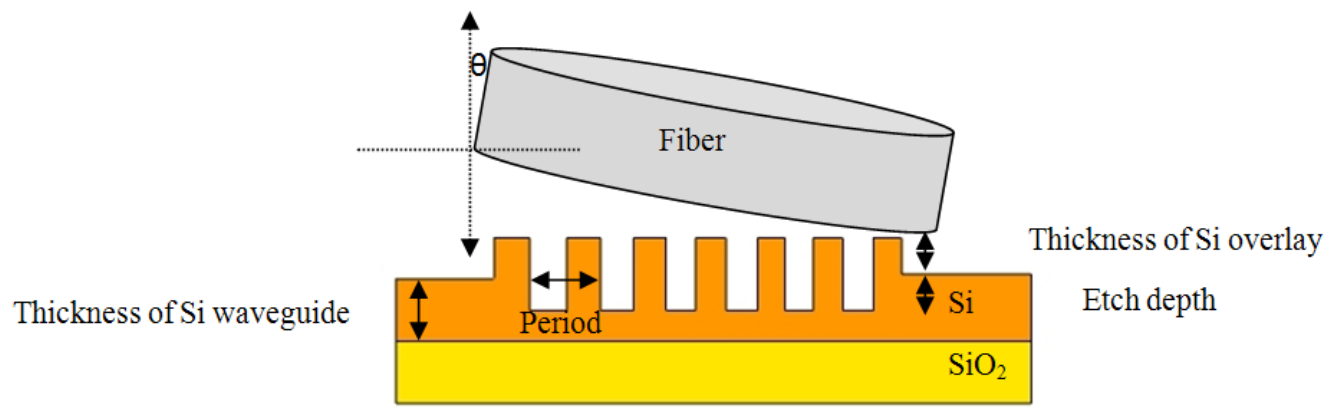

Fig.1 Schematic cross-section of the fiber-chip grating coupler interface

In the simulation, a 15 degree angle of the fiber is used to avoid $2^{\text {nd }}$ order reflection back into the waveguide. The etch depth is fixed at $70 \mathrm{~nm}$, the thickness of the Si overlay is $160 \mathrm{~nm}$ and the thickness of Si waveguide is $220 \mathrm{~nm}$. The period corresponding to a peak in the coupling efficiency at a wavelength of $2.25 \mu \mathrm{m}$ is $1.05 \mu \mathrm{m}$. The simulation itself is done in 2D using CAMFR, a full-vectorial solver based on eigen mode expansion. The simulated coupling efficiency is shown in Figure 2(a). It shows a maximum coupling efficiency around $-3.2 \mathrm{~dB}$ and a $3 \mathrm{~dB}$ bandwidth of $200 \mathrm{~nm}$. Figure 2 (b) shows an electric field plot obtained by simulation in Camfr (for TE polarized light at $2250 \mathrm{~nm}$ ).

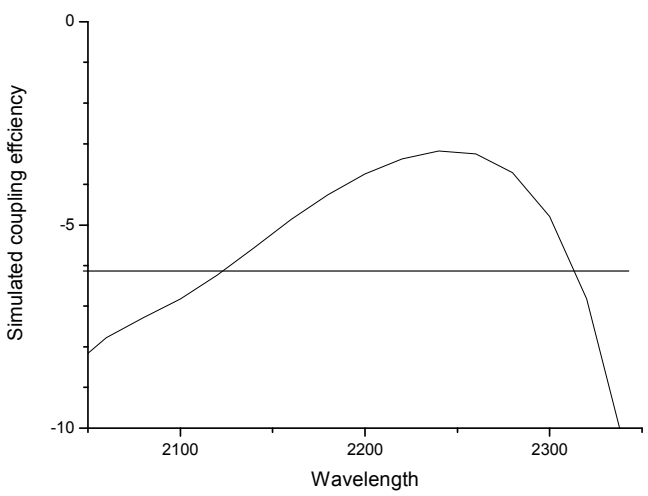

(a)

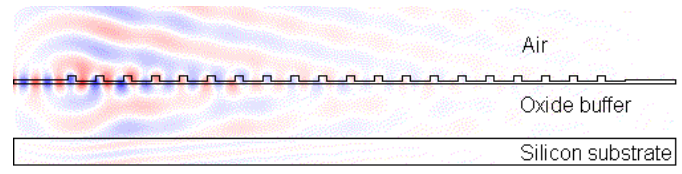

(b)

Fig.2 (a) Simulated coupling efficiency of the grating coupler (2D simulation) (b) Simulated field plot obtained from Camfr.

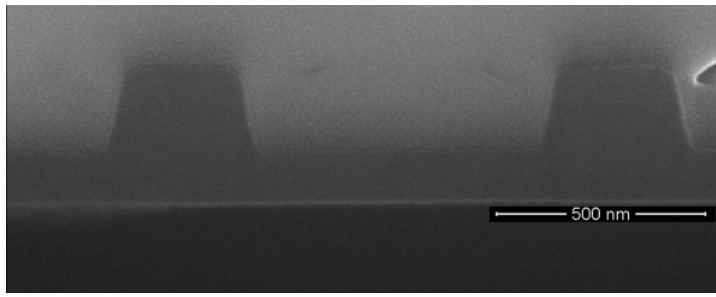

(a)

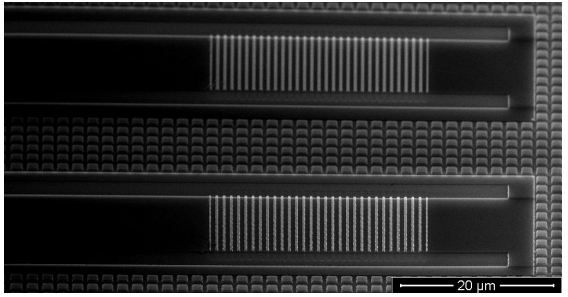

(b)

Fig. 3 (a) A cross-section of the grating (left) and (b) a bird eye top view of the fabricated raised grating (right)

\section{Experimental Results}

The fabrication of the highly efficient grating couplers is done in the $200 \mathrm{~mm}$ CMOS pilot line at imec with $193 \mathrm{~nm}$ DUV lithography. On top of a $200 \mathrm{~mm}$ SOI wafer consisting of a $220 \mathrm{~nm}$ thick crystalline silicon layer on top of a 2 um thick buried oxide layer, $160 \mathrm{~nm}$ of poly-crystalline silicon is deposited. To form the slits of the grating coupler, 
this stack is etched through the top poly-silicon layer and $70 \mathrm{~nm}$ through the crystalline silicon layer. Except at the location of the grating couplers the poly-silicon layer is removed with a chlorine/fluorine-based and bromine-based chemistry. We designed grating couplers with a period of $1.050 \mathrm{um}$. The fabricated couplers are shown in Figure 3. The grating duty cycle is $35 \%$. To determine the coupling efficiency, the fiber-to-fiber insertion loss of two grating couplers was measured. These grating couplers were connected by a low-loss waveguide of $300 \mathrm{um}$ long and $12 \mathrm{um}$ wide. We have characterized the gratings using standard single mode fibers under an angle of 15 degrees. The results are shown in figure 4 . The small reflections at the grating couplers cause Fabry-Perot fringes which can be seen, especially for the longer wavelengths, since we come closer to vertical diffraction, which induces a second order back reflection. The maximum coupling efficiency is found to be $-5.2 \mathrm{~dB}$ and the $3 \mathrm{~dB}$ bandwidth is $160 \mathrm{~nm}$. The lower than expected efficiency is believed to be a result of the lateral size of the grating. The grating is 12 um wide, which makes the overlap with the fiber suboptimal. The mode diameter in the fiber is calculated to be 13.6 um at $2.15 \mu \mathrm{m}$, which causes an extra loss of $1.5 \mathrm{~dB}$ compared to the optimal situation of where a 20 um wide grating coupler is used. The wavelength shift between theory and experiment is attributed to a mismatch between the simulated device parameters and the experimentally realized grating structure.

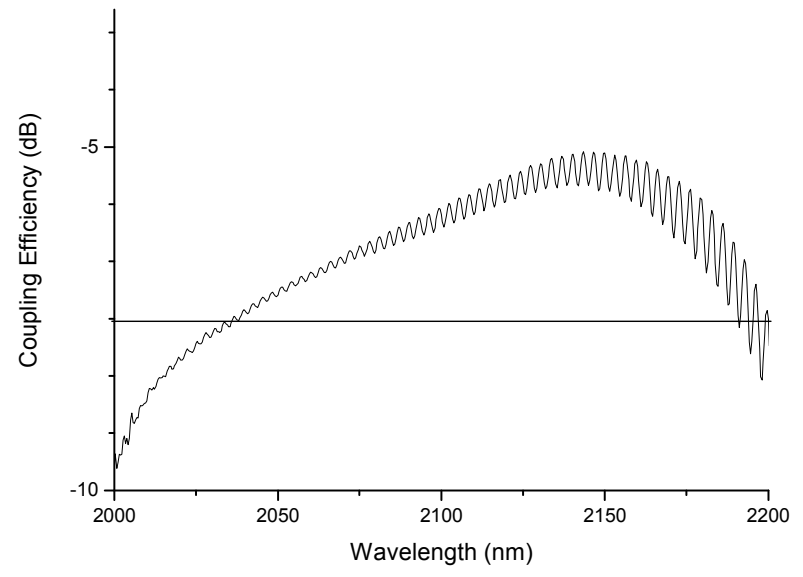

\section{Conclusions}

Figure 4: The fiber-chip coupling efficiency as a function of wavelength

We have demonstrated for the first time efficient grating couplers for the short-wave infrared wavelength range. A coupling efficiency of $-5.2 \mathrm{~dB}$ was obtained with a $160 \mathrm{~nm} 3 \mathrm{~dB}$ bandwidth. The gratings open the path to new silicon photonic chips for the mid-infrared enabling new nonlinear optical functions as well as new spectroscopic lab on-a-chip approaches. Further optimization to improve the efficiency will be discussed at the conference.

\section{Acknowledgements}

The authors would like to thank X. Liu and R. Osgood for many helpful discussions. They also would like to thank Shahram Keyvaninia for the FIB cross-section on the grating structure.

\section{References}

[1] W. Bogaerts et al., "Basic structures for photonic integrated circuits in Silicon-on-insulator," Opt. Express 12, 1583-1591 (2004)

[2] R. Soref, "Mid-infrared photonics in silicon and germanium", Nature Photonics 4, 495 - 497 (2010)

[3] Crowder et al., "Infrared methods for gas detection", in Mid-infrared semiconductor optoelectronics, Springer (2006)

[4] S. Zlatanovic et al., "Mid-infrared wavelength conversion in silicon waveguides using ultracompact telecom-band-derived pump source," Nature Photonics 4, 561-564 (2010).

[5] X. Liu et al., "Mid-infrared optical parametric amplifier using silicon nanophotonic waveguides," Nature Photonics 4, 557-560 (2010).

[6] B. Kuyken et al., "Generation of a telecom-to-mid-infrared spanning supercontinuum using silicon-on-insulator wire waveguides",submitted for publication at CLEO 2011, 